\title{
Serum ghrelin and obestatin levels in patients with acne vulgaris: are they important for the severity?
}

\author{
Zekiye Kanat ${ }^{1}$, İbrahim Kökçam² ${ }^{2}$ Musa Yılmaz ${ }^{3}$, Süleyman Aydın², Zeynep Özkan \\ ${ }^{1}$ Dermatology Department, Elazığ Training and Research Hospital, Elazığ, Turkey \\ 2Dermatology Department, Faculty of Medicine, Adıyaman University, Elazığ, Turkey \\ ${ }^{3}$ Biochemistry Department, Faculty of Medicine, Firat University, Elazığ, Turkey \\ ${ }^{4}$ General Surgery Department, Elazığ Training and Research Hospital, Elazığ, Turkey
}

Adv Dermatol Allergol 2019; XXXVI (4): 412-418

DOI: https://doi.org/10.5114/ada.2019.87445

\begin{abstract}
Introduction: Acne vulgaris (AV) is a chronic, inflammatory disease of the pilosebaceous unit. Recently, three peptide-structured hormones, products of a single gene, have been discovered. These hormones are acylated ghrelin, des-acyl ghrelin and obestatin.

Aim: To demonstrate the association of serum acylated ghrelin, des-acyl ghrelin, and obestatin levels with acne severity.

Material and methods: A total of 63 patients grouped as mild $(n=22)$, moderate $(n=21)$ and severe $(n=20)$ acne according to the Global Acne Grading System and 20 medically healthy volunteers were included in the study. Serum ghrelin and obestatin levels obtained from the participants were examined.

Results: When mean ghrelin, des-acyl-ghrelin and obestatin values of the acne-group (AG) were compared with the control group (CG), they were found be lower in the AG, but were not statistically significant. Among the patient groups, while acylated ghrelin values were highest in the severe AG, des-acyl ghrelin values were highest in mild severe $A G$ and mean obestatin values were highest in moderate severe $A G(p>0.05)$. When the groups were compared for obestatin values; the highest average value was detected in the CG. However, it was not significant when the groups were compared.

Conclusions: It has been suggested that there may be a link between acne and the levels of acylated ghrelin, desacyl ghrelin and obestatin which are decreased in the serum of acne patients. Because of the decrease observed in the levels of these hormones which have antimicrobial features, we suggest that inflammation in acne cannot be suppressed and the reproduction of the microorganisms that play a role in the aetiology of the disease cannot be prevented. The replacement of these hormones at physiologic concentrations may contribute to the acne treatment.
\end{abstract}

Key words: acne vulgaris, ghrelin, des-acyl ghrelin, obestatin.

\section{Introduction}

Acne vulgaris (AV) is a chronic, inflammatory disease of the pilosebaceous unit. It can cause serious limitations in individuals, in terms of social and psychological functions, although it is thought to mostly cause cosmetic complaints. Its prevalence reaches up to $80 \%$. Although it commonly begins during the pubertal period, it may begin in the post pubertal period, too [1, 2].

Although its etiopathogenesis is unclear, recent studies demonstrate that it is multifactorial. 4 principal factors that play a role in acne development are: follicular ductal hypercornification, increase in sebum secretion, follicular Propionibacterium acnes (P. acnes) colonization and inflammation. It is still unclear which mechanisms initiate inflammation. Comedone formation was at first thought not to be an inflammatory event but it has been demonstrated in later immunohistochemical studies that microcomedones are the earliest steps of the inflammatory acne lesions [1-3].

There are many studies that associate hormones with $\mathrm{AV}$ [4]. Androgens are among the most studied hormones known to affect cutaneous biology. The roles of androgens in sebum production are yet to be known. However, many studies performed demonstrated that human pilosebaceous unit can be under the effect of several hor-

Address for correspondence: Zekiye Kanat MD, Dermatology Department, Training and Research Hospital, Elazı̆̆, Turkey, phone: +90 424 2381000-1408, e-mail: zekiye-er-kanat@hotmail.com Received: 1.01.2018, accepted: 13.04.2018. 
mones consisting amino acid, oligopeptide, polypeptide, protein, glycoprotein, lipid and phospholipid structures besides the androgens [5].

Recently, three peptide-structured hormones, product of a single gene, have been discovered. These hormones are acylated ghrelin, des-acyl ghrelin and obestatin. Initially it has been postulated that des-acyl ghrelin has no biological effect, later on it was reported that both acylated and des-acyl ghrelin have roles in the release of the growth hormone and functions on the regulation of appetite. Besides all these major effects, it has been reported that ghrelin has anti-inflammatory and antimicrobial effects. Although the initial studies on obestatin, which is coded by the same gene as ghrelin, proposed that obestatin has a contrast effect on ghrelin, the obestatin/ ghrelin relationship is still unclear [6-8].

\section{Aim}

The aim of the study is to demonstrate the association of serum acylated ghrelin, des-acyl ghrelin, and obestatin levels with acne severity.

\section{Material and methods}

This study was initiated with the approval from the Firat University Dean of the School of Medicine Ethics Committee on 12.08.2011 and decision No. 12/12.

We planned our work to include 30 people in each group and a healthy control group. However, some people withdrew from the study with time so that the study was performed with total 63 patients that came to the outpatient clinic between January 2012 and September 2012 with the complaint of AV and 20 healthy volunteers. The patients and volunteers included in the study were informed and consent was received.

\section{Study plan}

In this study, the acne groups (AG) were created from the patients who were diagnosed as AV according to "Acne vulgaris: review and guidelines 2009" [9] and had the following characteristics:

- Age of 18-30 years,

- Having normal body mass index (BMI),

- Not on any diet,

- No pregnant,

- Having no endocrinologic disease such as diabetes, hyper- or hypothyroidism,

- Having no history of cancer,

- Not using alcohol,

- Not receiving systemic medication treatment and those who accepted to voluntarily participate in the study.

The control group (CG) consisted of healthy individuals with similar BMI, age range and not using alcohol or any medications. The CG was selected according to similar characteristics in the AG.
The detailed histories of the patients included in the study groups were taken and their physical examinations were performed. Gender, age, height and weight were recorded in the study forms. In the patient groups, diagnosis and determination of acne severity were performed by the same physician. In the case groups, the severity of the disease was evaluated according to the Global Acne Grading System (GAGS) and

- Patients with scores $\leq 18$ were classified as mild severe,

- Patients with scores between 19-30 were classified as moderate severe and

- Patients with scores $\geq 31$ were classified as severe acne.

Body mass index was calculated. For calculation of $\mathrm{BMI}$, weight $[\mathrm{kg}] /$ height $\left[\mathrm{cm}^{2}\right]$ formula was used [10]. For calculating insulin resistance, homeostasis model assessment of insulin resistance (HOMA-IR), glucose $[\mathrm{mg} / \mathrm{dl}]$ $\times$ insulin $[\mathrm{U} / \mathrm{ml}] / 405$ formula was used [11].

\section{Measurement of serum ghrelin, des-acyl ghrelin and obestatin levels}

Serum ghrelin and obestatin levels were measured using the Enzyme Linked Immunosorbent Assay (ELISA) method with commercial kits. Since these peptide hormones can be degraded by proteases, in order to prevent proteolysis, 500 kallikrein unit of aprotinin per millimetre was added to plain biochemistry tubes before obtaining blood samples from the participants. $5 \mathrm{ml}$ fasting blood samples in the morning were obtained from each participant and centrifuged at 3000 rpm for 5 min and then the obtained sera were transferred to serum Eppendorf tubes and stored in the deep freezer at $-20^{\circ} \mathrm{C}$ until the day of the study.

After the collection of samples from the patients was completed, ghrelin vs obestatin levels were measured in the collected serum samples by ELISA. Human ghrelin vs obestatin kits specifically produced for humans were used for this purpose. To measure acylated ghrelin and des-acyl ghrelin SPI-BIO mark kit (A05119: Unacylated Ghrelin (human) manufactured by Bertin Pharma EIA kit vs. A05106: Acylated Ghrelin (human) ElA kit) were used. For measuring obestatin, human-obestatin ELISA-kit (Catalogue Number CSB-E-12818-h) of Cusabio Biotech was used.

\section{Ethics}

This study was initiated with the approval from the Firat University Dean of the School of Medicine Ethics Committee on 12.08.2011 and decision No. 12/12.

\section{Statistical analysis}

The data obtained from the study were presented as mean $\pm S D$. Student- $t$ test was used to compare the CG and $A G$ and to compare the measured values within the 
AG; $\chi^{2}$ test was used in order to compare the values of $\mathrm{CG}$ and $\mathrm{AG}$ obtained by counting. Chi-square and Kolmogorov Smirnov tests were used to compare data obtained by counting the acne group. Also, variance analysis test was used for the comparison of group averages. For all tests, $p<0.05$ value was accepted as statistically significant criterion.

\section{Results}

When sixty three patients were classified according to GAGS, 22 (34.9\%) cases were mild, 21 (33.3\%) cases were moderate and 20 (31.7\%) cases were evaluated as severe acne. There was statistically no significant difference for gender distribution between the groups $(p>0.05)$.

The age range was 18-29 years in the mild and moderate groups, 18-24 years in the severe group and 18-29 years in CG. The mean age of the mild group was 21.18 \pm 2.7 years; the mean age of the moderate group was $21.09 \pm 2.6$ years; the mean age of the severe group was $20.71 \pm 2.1$ years and mean age of the CG was $23.8 \pm 3.42$ years. Demographic characteristics of $A G$ and $C G$ were given in Table 1.

Acylated ghrelin mean values of mild, moderate and severe groups were close to each other and were not statistically significant $(p>0.05)$. Although there was no statistically significant difference found between serum ghrelin levels of patients with severe and mild acne, these values were detected to be higher in the severe acne group.

When the AG was compared with the CG; acylated ghrelin levels of AG were relatively lower than in the CG but the difference was not statistically significant $(p>$ 0.05). When AG were compared between themselves and with the CG, in terms of des-acyl ghrelin levels, the lowest average was detected in the moderate severe acne group and the highest average value was measured in the CG. However, these differences were not statistically significant $(p>0.05$ and $p>0.05)$. When the groups were compared for obestatin values, the highest average value was detected in the CG. However, it was not significant when the groups were compared $(p>0.05$ and $p>0.05)$ (Table 2 and Figures 1-3).

Similarly, in terms of HOMA-IR and BMI, there was no significant difference when AG and CG were compared. There was also no significance detected when the AG were compared between themselves (Table 3).

\section{Discussion}

Acne vulgaris is a chronic, inflammatory disease of the pilosebaceous unit. The disease is common in adolescents and mostly thought to cause cosmetic problems; it also may cause serious restrictions in terms of psychological and social well-being. AV frequency varies from society to society, in America its frequency is $13-16 \%$ between the ages of 15 to 44 [1-4]. In studies conducted in our country its frequency is reported as $16-40 \%$ between the ages of 14 and 19, 10-20\% in the paediatric age group and $3-5 \%$ between the ages of 40 and $49[12,13]$.

The skin, particularly the pilosebaceous unit, can be accepted as an endocrine organ where several hormones and receptors are synthesized. Recently many studies have been conducted on the roles of hormones such as

Table 1. Demographic characteristics of the acne and control groups

\begin{tabular}{|c|c|c|c|c|c|c|}
\hline \multirow[t]{2}{*}{ Parameter } & \multicolumn{4}{|c|}{ Acne group (AG) } & \multirow{2}{*}{$\begin{array}{l}\text { Control group } \\
\text { (CG) }\end{array}$} & \multirow[t]{2}{*}{$P$-value } \\
\hline & Mild & Moderate & Severe & Total & & \\
\hline$N$ & 22 & 21 & 20 & 63 & 20 & $>0.05$ \\
\hline$\%$ & 34.9 & 33.3 & 31.7 & 75.9 & 24.1 & $>0.05$ \\
\hline Gender (M/F) & $12 / 10$ & $11 / 11$ & $9 / 11$ & $32 / 31$ & $12 / 8$ & $>0.05$ \\
\hline $\begin{array}{l}\text { Age, mean } \pm \text { standard } \\
\text { deviation [year] }\end{array}$ & $21.18 \pm 2.7$ & $21.09 \pm 2.6$ & $20.71 \pm 2.1$ & $21.03 \pm 2.9$ & $23.8 \pm 3.42$ & - \\
\hline
\end{tabular}

Table 2. Acylated ghrelin, des-acyl ghrelin and obestatin values of the acne and control groups

\begin{tabular}{|c|c|c|c|c|c|c|}
\hline \multirow[t]{2}{*}{ Parameter } & \multicolumn{4}{|c|}{ Acne group (AG) } & \multirow{2}{*}{$\begin{array}{l}\text { Control group } \\
\text { (CG) }\end{array}$} & \multirow[t]{2}{*}{$P$-value } \\
\hline & Mild & Moderate & Severe & Total & & \\
\hline Acylated ghrelin & $29.51 \pm 5.79$ & $29.40 \pm 11.08$ & $32.43 \pm 11.14$ & $30.54 \pm 9.72$ & $33.53 \pm 10.67$ & 0.24 \\
\hline Des-acyl ghrelin & $384.21 \pm 256.29$ & $339.32 \pm 191.91$ & $366.76 \pm 216.53$ & $363.61 \pm 224.07$ & $436.90 \pm 249.45$ & 0.21 \\
\hline Obestatin & $76.19 \pm 48.47$ & $84.28 \pm 54.12$ & $64.64 \pm 33.35$ & $76.08 \pm 46.74$ & $91.49 \pm 30.00$ & 0.17 \\
\hline
\end{tabular}

Results presented as mean \pm standard deviation. 


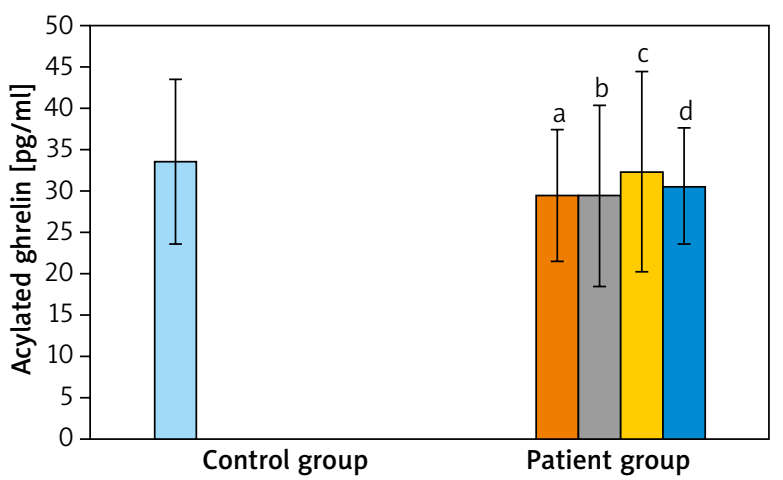

Figure 1. Acylated ghrelin: $a$ - mild, $b$ - moderate, $c$ - severe, $d$ - total

steroid hormones, phospholipid hormones, polypeptide and protein structured corticotropin-releasing hormone, adrenocorticotropic hormone, and melanocyte-stimulating hormone [5].

Recently a few hormones coded by the same gene have been discovered (obestatin, des-acyl ghrelin and acylated ghrelin that is also known as a bioactive peptide). The mRNA of ghrelin has been detected in almost all tissues. In the studied tissues, ghrelin mRNA levels were detected maximum in the stomach fundus, it exists in many tissues including the skin and has an effect on many systems in humans $[6,7]$. Although ghrelin basically functions by increasing the release of the growth hormone, it also plays a role in food intake, carbohydrate metabolism, adipogenesis, reproduction and cell proliferation $[6,7,14]$. Studies have shown that ghrelin has an anti-inflammatory property. Besides, it has been stressed that the hormones coded by the ghrelin gene are related with inflammation as well. Although there are studies that reported obestatin as especially having an opposite effect to ghrelin, the obestatin-ghrelin relation is yet unclear [7, 14].

Although the role of obestatin discovered by Zhang et al. [14] is not fully understood, it is known that obestatin levels are not affected by fasting or postprandial state. Obestatin is an anorectic peptide and suppresses weight gain. Although it is the same gene product as ghrelin, the studies on obestatin are limited. For this reason, very little is known about the physiologic role of obestatin in humans [15].

The studies conducted on ghrelin and ghrelin gene products: obestatin and des-acyl ghrelin have shown that

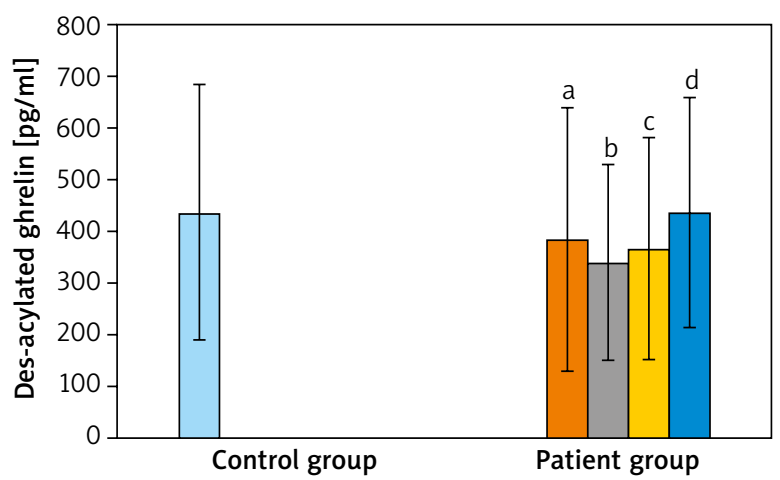

Figure 2. Des-acylated ghrelin: $a$ - mild, $b$ - moderate, $c$ - severe, $d$ - total

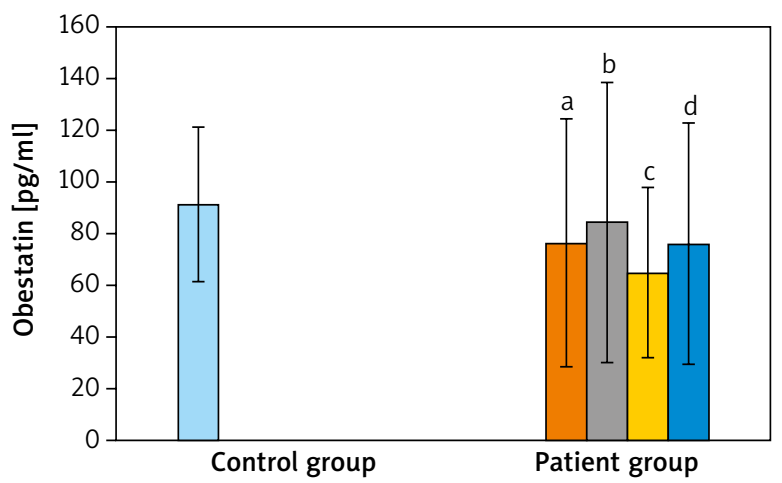

Figure 3. Obestatin: $a$ - mild, b-moderate, c - severe, d-total

these hormones have roles in many diseases as well as their treatment. Several studies have been conducted about the relationship of ghrelin with diabetes, cancer, polycystic ovary disease, hypertension, hepatic diseases, endocrine diseases and inflammatory diseases. The studies on these hormones and skin diseases are very few and the relation of these hormones and the skin is intriguing $[5,7,14,15]$.

Although the main cause of the disease is unknown, it is accepted that inflammation has an important role in acne pathogenesis. For this reason, many researchers showed interest in looking into the factors initiating inflammation and serum markers that are indicators of inflammation [3].

This study aimed to reveal whether obestatin and ghrelin hormones have roles in the etiopathogenesis of acne and to examine the levels of these hormones in

Table 3. Body mass index (BMI) and homeotasis model assessment of onsulin resistance (HOMA-IR) values of the acne and control groups

\begin{tabular}{|c|c|c|c|c|c|}
\hline Parameter & Mild & Moderate & Severe & Control group (CG) & $P$-value \\
\hline HOMA-IR & $1.78 \pm 1.41$ & $1.35 \pm 1.15$ & $1.16 \pm 1.05$ & $1.90 \pm 1.59$ & 0.2 \\
\hline $\mathrm{BMI}$ & $20.97 \pm 2.31$ & $21.37 \pm 1.91$ & $21.98 \pm 2.13$ & $21.75 \pm 2.56$ & 0.3 \\
\hline
\end{tabular}

Results presented as mean \pm standard deviation. 
acne. As a result of the evaluation, acylated ghrelin, desacyl ghrelin and obestatin levels were seen to be lower in the AG than the CG, although there was no statistically significant difference among these groups.

There are several studies in order to demonstrate the role of ghrelin in proinflammatory diseases conducted on humans and animals [16]. Increased serum or plasma ghrelin levels have been demonstrated in many inflammatory diseases including ulcerative colitis, Crohn's disease, ankylosing spondylitis, sepsis, pancreatitis and colitis. It has been demonstrated that in these diseases there is a correlation between increased serum and plasma ghrelin levels with severity of disease and at the same time the correlation has been demonstrated between ghrelin levels and proinflammatory cytokines, particularly tumor necrosis factor $\alpha$ (TNF- $\alpha$ ), interleukin (IL)-6, IL-1 $1 \beta$ expressions [17]. According to this, in acne as the inflammatory process increases, an increase in ghrelin levels was expected. However, in our study although there was no statistically significant difference found between the groups for ghrelin levels, there were higher values of these hormones in the CG. Based on this, these hormones are suggested to be insufficient in suppressing inflammation in acne.

Koca et al. [18] have evaluated the relationship between disease activity and serum ghrelin and obestatin levels in rheumatoid arthritis and Behçet's disease patients in their study. As a result of their study, although no correlation between total ghrelin levels and any inflammatory parameter was observed, they have demonstrated a correlation between obestatin levels which is coded by the same gene as ghrelin and some inflammatory parameters. Eventually they proposed that serum ghrelin levels in rheumatoid arthritis may be decreased due to cytokines or inflammatory process and therefore did not have a correlation with inflammation indicators [18].

Peracchi et al. [19] have evaluated ghrelin levels in inflammatory bowel diseases. They have compared ghrelin levels of active inflammatory bowel disease patients and patients with inflammatory bowel disease in remission. They detected abnormally high ghrelin levels in active inflammatory bowel disease patients as compared to patients in remission. Again in another study conducted by Peracchi et al. [20], a correlation was demonstrated between high levels of ghrelin in patients with newly diagnosed active Celiac disease and severity of intestinal mucosal lesions with these high levels of ghrelin. They also detected a decrease in the normal level of ghrelin in patients successfully treated with gluten-free diet [20]. Consequently, they emphasized the probable role of ghrelin in the inflammatory process of inflammatory bowel diseases. They also suggested that some inflammatory mediators and products released during the disease process may cause the stimulation of ghrelin release. They stressed that normal, low as well as high levels of ghrelin could be detected in inflammatory diseases [19].
A positive correlation between the severity of acne and severity of inflammation has been known for a long time [3]. In our study, although there was no statistically significant difference found between serum ghrelin levels of patients with severe and mild acne, these values were detected to be higher in severe AG. Since inflammation in acne occurs locally without causing systemic findings, the inflammatory mediators are released locally at the disease site [3]. For this reason, ghrelin levels may be decreased due to either the type of the mediator released or localized inflammation. In this context, we believe that studies on the level of ghrelin at the tissue level where the development of inflammation is prominent, can provide more satisfactory results about the roles of hormones in acne development.

The relationship of ghrelin with the inflammatory process has also been evaluated experimentally. In a study of Kerem et al. [21] performed on 90 rats, they compared experimentally created mild pancreatitis and severe pancreatitis in groups of rats and reported that ghrelin levels may have increased during the inflammatory process. Although our study was a human study, it is also different from this study in terms of the time the hormone levels were studied. In this study, serum ghrelin levels were studied not at the beginning of the disease process but at different stages of the disease. Therefore we believe that it would be more significant to study pretreatment and post-treatment ghrelin levels in the future.

Another research team again evaluated the relationship of the severity of acute pancreatitis and serum ghrelin concentrations performed on patients with pancreatitis and detected high serum ghrelin levels during the application in the risky group. However, they reported no significant differences between the two groups after $48 \mathrm{~h}$ and discharge [22]. In both studies, blood samples were obtained at the beginning of the study. In our study, the patients were included into the study not at the beginning, but during the chronic period. Measured ghrelin and obestatin levels were found to be higher in the control group than the case group. In fact, an increase in ghrelin levels was expected during the inflammatory process. In many studies, such values were detected. However, as in our study, ghrelin levels were detected to be low as the blood samples were obtained during the chronic process of the disease. This suggests that, since ghrelins have anti-inflammatory properties, they should be decreased because they were used up in the biochemical pathways to eliminate inflammation. Also, because ghrelins have antioxidant features, we suggested that its amounts may decrease in order to eliminate the disease burden. Our findings and other research findings suggest that ghrelin levels differ in the disease periods and it would be beneficial to take into consideration in which disease period ghrelin levels were studied.

As known, there are many factors involved in the development of acne. One of them is microorganisms. The 
major microorganisms are P. acnes, Malassezia furfur and Staphylococcus epidermidis [23]. Also it has been reported in some studies that $S$. aureus also has a role in acne development $[24,25]$. Previous studies demonstrated that ghrelin has antimicrobial effects, for example: with the exception of Gram-negative bacteria such as Escherichia coli and Pseudomonas aeruginosa it was also reported to prevent the reproduction of Gram-positive bacteria such as Staphylococcus aureus (S. aureus) and Enterococcus faecalis [26]. Ghrelin which is researched in this study is considered to have an antimicrobial effect on S. aureus and the low levels of ghrelin detected may suggest that acne was developed due to the decreased ghrelin levels that could not suppress the reproduction of S. aureus. However, this hypothesis is can only be validated after confirmation in animal experiments on whether there was a decrease or increase in ghrelin hormone concentrations in skin scrapings of the animals, based on this we believe that the role of ghrelin in the treatment of acne will be better ascertained.

It is known that $P$. acnes are more important in the etiopathogenesis of acne than $S$. aureus. We did not come across any study on whether or not the ghrelin hormone has an antimicrobial effect on $P$. acnes. When we considered previous studies, ghrelin has an antimicrobial effect on resistant microorganisms such as $S$. aureus, thus we suggested that it will probably be effective on $P$. acnes as well. Therefore, we suggested that researching the antimicrobial effects of ghrelin on $P$. acnes and the evaluation of the relationship of ghrelin concentrations and reproduction of this bacteria will provide contribution to the aetiology and treatment of acne.

Bresciani et al. [27] have demonstrated that in vivo obestatin does not change the growth hormone $(\mathrm{GH})$ and corticosterone secretion. In the study that Yamamoto et al. [28] performed on rats, they demonstrated that obestatin does not affect plasma GH, PRL, ACTH and TSH levels. According to these data, it is seen that obestatin has no relation with the hormone levels known to be related with the pathogenesis of acne [15, 27, 28].

In a study of Ceranowicz et al. [29], they created acute pancreatitis on rats by giving caerulein, evaluated whether obestatin has a protective effect on pancreatitis by administering an intraperitoneal obestatin injection. As a result, they detected that obestatin decreases the severity of acute pancreatitis due to caerulein. Koca et al. [18] examined the serum obestatin levels in rheumatoid arthritis and reported that obestatin is correlated with some inflammatory markers in serum. Eventually it was emphasized that obestatin is more valuable than ghrelin in the pathogenesis of rheumatoid arthritis. In our study, while the mean obestatin level of the case group was $76.08 \pm 46.74$, it was $91.49 \pm 30.00$ in the CG. Based on these findings, lower obestatin values in the acne group suggest that it may be used up in inflammatory pathways and thus decrease.
Body mass index is the most important parameter used to evaluate obesity. Tsai et al. [30] have reported in their study that high BMI in school children is a prominent risk factor for acne. They detected that in most cases, the lesions were activated with fatty foods; nutrition with high glycaemic index increases acne development via sex hormones and insulin-like growth factor-1 (IGF-1) [31]. Since the cases with normal BMI were included in our study, there was no relationship detected between $\mathrm{BMI}$ and the severity of acne.

Homeostasis model assessment of insulin resistance is a parameter used for determining insulin resistance. In a study that compared the acne group fed with proteinand carbohydrate-rich foods, there was no significant difference found between the two groups for severity of inflammation with HOMA-IR values [32]. In our study, the patient groups were created independently from dietary habits, similar to this study, there was no any relationship found between HOMA-IR and severity of acne.

According to the current literature on acne and ghrelin studies, ghrelin produced in deep hair follicles and sebaceous glands may fold pathogenesis of AV and cause AV in humans to decrease serum ghrelin [33]. In a study evaluating adipokines in patients with AV again, ghrelin may be the last to fail to participate in responsible mechanisms in non-obese patients with moderate AV [34]. In addition, isotretinoin used in acne treatment increased leptin and adiponectin levels and showed anti-inflammatory activity but not the ghrelin level [35].

\section{Conclusions}

Apart from the increase in androgen sensitivity, sebum secretion, microorganisms and inflammation that play roles in the aetiology of AV, we think that decreased acylated ghrelin, des-acyl ghrelin and obestatin levels also play roles in the pathogenesis. Because of the observed decrease in levels of these hormones that have antimicrobial features, we predicted that the inflammation in acne cannot be suppressed and reproduction of the microorganisms that play important roles in the aetiology of the disease cannot be prevented; therefore replacement of these hormones to physiologic concentrations may contribute to the treatment of acne.

\section{Acknowledgments}

This study was presented as a poster at the $25^{\text {th }} \mathrm{Na}$ tional Dermatology Congress, 21-25 Nov 2014, Antalya, Turkey.

This work was funded by the Frrat University Scientific Project Unit (FÜBAP).

\section{Conflict of interest}

The authors declare no conflict of interest. 


\section{References}

1. Zaengllein AL, Thiboutot DM. Acne vulgaris. In: Dermatology. Bolognia JL, Jorizzo JL, Rapini RP (eds.). $2^{\text {nd }}$ edn. Mosby Elsevier, New York 2008; 495-508.

2. Rzany B, Kahl C. Epidemiology of acne vulgaris. J Dtsch Dermatol Ges 2006; 4: 8-9.

3. Farrar MD, Ingham E. Acne: inflammation. Clin Dermatol 2004; 22: 380-4.

4. Kaymak Y, Adısen E, Ilter N, et al. Dietary glycemic index and glucose, insulin, insulin-like growth factor-1, insulin-like growth factor binding protein 3 and leptin levels in patients with acne. J Am Acad Dermatol 2007; 57: 819-23.

5. Chen WC, Zouboulis CC. Hormones and the pilosebaceous unit. Dermatoendocrinol 2009; 1: 81-6.

6. Hirayama T, Kawabe T, Matsushima M, et al. Ghrelin and obestatin promote the allergic action in rat peritoneal mast cells as basic secretagogues. Peptides 2010; 31: 2109-13.

7. Aydin S, Erenler SO, Kendir Y. Effects of sodium octanoate, acylated ghrelin and desacylated ghrelin on the growth of genetically enginneered Escherichia coli. J Med Biochem 2011; 30: 328-33.

8. Chorny A, Anderson P, Gonzalez-Rey E, Delgado M. Ghrelin protects aganist experimental sepsis by killing bacteria. J Immunol 2008; 180: 8369-77.

9. Ramos-e-Silva M, Carneiro SC. Acne vulgaris: review and guidelines. Dermatol Nurs 2009; 21: 63-8.

10. Sterry W, Strober BE, Menter A. Obesity in psoriasis: the metabolic, clinical and therapeutic implications. report of an interdisciplinary conference and review. Br J Dermatol 2007; 157: 649-55.

11. Kondo N, Nomura M, Nakaya Y, et al. Association of inflammatory marker and highly sensitive $C$ reactive protein with aerobic exercise capacity, maximum oxygen uptake and insulin resistance in healthy middle aged volunteers. Circ J 2005; 69: 452-7.

12. Akyol M, Özçelik S. Epidemiology of acne vulgaris. Turkiye Klinikleri J Dermatol-Special Topics 2009; 2: 6-10.

13. Tamer E, ilhan MN, Polat M, et al. Prevalence of skin dis eases among pediatric patients in Turkey. J Dermatol 2008; 35: 413-8.

14. Zhang JV, Ren PG, Avsian-Kretchmer O, et al. Obestatin, a peptide encoded by the ghrelin gene, opposes ghrelins effects on food intake. Science 2005; 310: 996-9.

15. Lacquaniti A, Donato V, Chirico V, et al. Obestatin: an interesting but controversial gut hormone. Ann Nutr Metab 2011; 59: 193-9.

16. Dixit VD, Schaffer H, Pyle R, et al. Ghrelin inhibits leptin and activation-induced proinflammatory cytokine expression by human monocytes and T cells. J Clin Invest 2004; 114: 57-66.

17. Baatar D, Patel K, Taub DD. The effects of ghrelin on inflammation and immune system. Mol Cell Endocrinol 2011; 340: 44-58.

18. Koca SS, Ozgen M, Aydın S, et al. Ghrelin and obestatin levels in rheumatoid arthritis. Inflammation 2008; 31: 329-35.

19. Peracchi M, Bardella MT, Caprioli F, et al. Circulating ghrelin levels in patients with inflammatory bowel disease. Gut 2006; 55: 432-3.

20. Peracchi M, Conte D, Terrani C, et al. Circulating ghrelin levels in celiac patients. Am J Gastroenterol 2003; 98: 2474-8.

21. Kerem M, Bedirli A, Pasaoglu H, et al. Role of ghrelin and leptin in predicting the severity of acute pancreatitis. Dig Dis Sci 2007; 52: 950-5.
22. Lee SH, Kim YD, Kong YH, et al. The relevance of serum ghrelin concentration to severity of acute pancreatitis. Gut Liver 2010; 4: 234-40.

23. Oberemok SS, Shalita AR. Acne vulgaris. I: pathogenesis and diagnosis. Cutis 2002; 70: 101-5.

24. Hassanzadeh P, Bahmani M, Mehrabani D. Bacterial resistance to antibiotics in acne vulgaris: an in vitro study. Indian J Dermatol 2008; 53: 122-4.

25. Khorvash F, Abdi F, Kashani HH, et al. Staphylococcus aureus in acne pathogenesis: a case-control study. N Am J Med Sci 2012; 4: 573-6.

26. Min C, Ohta K, Kajiya M, et al. The antimicrobial activity of the appetite peptide hormone ghrelin. Peptides 2012; 36: 151-6.

27. Bresciani E, Rapetti D, Donà F, et al. Obestatin inhibits feeding but does not modulate $\mathrm{GH}$ and corticosterone secretion in the rat. J Endocrinol Invest 2006; 29: 16-8.

28. Yamamoto D, Ikeshita N, Daito R, et al. Neither intravenous nor intracerebroventricular administration of obestatin affects the secretion of GH, PRL, TSH and ACTH in rats. Regul Pept 2007; 138: 141-4.

29. Ceranowicz P, Warzecha Z, Dembinski A, et al. Pretreatment with obestatin inhibits the development of cerulein-induced pancreatitis. J Physiol Pharmacol 2009; 60: 95-101.

30. Tsai MC, Chen W, Cheng YW, et al. Higher body mass index is a significant risk factor for acne formation in schoolchildren. Eur J Dermatol 2006; 16: 251-3.

31. Smith R, Mann N, Makelainen $\mathrm{H}$, et al. A pilot study to determine the short term effects of a low glycemlic load diet on hormonal markers of acne: a non randomized paralel, controlled feding trial. Mol Nutr Food Res 2008; 52: 718-26.

32. Smith R, Mann N, Makelainen $\mathrm{H}$, et al. The effect of shortterm altered macronutrient status on acne vulgaris and biochemical markers of insulin sensitivity. Asia Pacific J Clin Nutr 2004; 13: 567.

33. Cicek D, Demir B, Erden I, et al. Ghrelin in the pilosebaceous unit: alteration of ghrelin in patients with acne vulgaris. Eur J Dermatol 2015; 254: 323-8.

34. Ozuguz P, Kacar SD, Asik G, et al. Evaluation of leptin, adiponectin, and ghrelin levels in patients with acne vulgaris. Hum Exp Toxicol 2016; 36: 3-7.

35. Cemil BC, Ayvaz HH, Ozturk G, et al. Effects of isotretinoin on body mass index, serum adiponectin, leptin, and ghrelin levels in acne vulgaris patients. Adv Dermatol Allergol 2016; 33: 294-9. 